\section{P2-331 NON-STEROIDAL ANTI-INFLAMMATORY DRUG AND ASPIRIN USE AND THE RISK OF HEAD AND NECK CANCER: A SYSTEMATIC REVIEW}

doi:10.1136/jech.2011.142976k.63

\section{P2-333 ACUTE MYOCARDIAL INFARCTION: INCIDENCE AND 30-DAY CASE FATALITY IN ENGLAND FOR FIRST AND RECURRENT EVENTS}

doi:10.1136/jech.2011.142976k.65

J C Wilson, ${ }^{*}$ L Anderson, L Murray, C Hughes. Queen's University Belfast, Belfast, UK

Introduction Use of non-steroidal anti-inflammatory drugs (NSAIDs) has been associated with a reduced risk of several cancers. We conducted a systematic literature review to investigate the association between NSAID/aspirin use and risk of head and neck cancer (HNC).

Method Medline, Embase, PubMed, Cochrane Library and Web of Science were systematically searched using terms for NSAIDs/ aspirin, HNC and observational/intervention study designs.

Results Of 9268 articles identified, two population-based prescribing database studies and three case-control studies met the selection criteria. The studies investigated different HNC sites. Only one study found a significant protective effect of aspirin use for HNC risk (OR $0.75,95 \%$ CI 0.58 to 0.96 ) and one showed a significantly increased risk of oral/oropharangeal cancer with non-low-dose aspirin NSAID use (OR 3.5, 95\% CI 1.8 to 6.7).

Conclusion No definitive conclusion on the effect of NSAIDs/aspirin on HNC risk was possible. Further robust large-scale studies are required to clarify any possible association.

\section{P2-332 SELECTING PRIORITY HEALTH RISK FACTORS FOR RESEARCHING PREVENTIVE INTERVENTIONS: A NEW ZEALAND EXAMPLE}

doi:10.1136/jech.2011.142976k.64

${ }^{1} \mathrm{~N}$ Wilson, ${ }^{*}{ }^{1} \mathrm{~T}$ Blakely, ${ }^{1} \mathrm{R}$ Foster, ${ }^{1} \mathrm{D}$ Hadorn, ${ }^{2} \mathrm{~T}$ Vos. ${ }^{1}$ University of Otago, Wellington, Wellington, New Zealand; ${ }^{2}$ University of Queensland, Brisbane, Australia

Introduction To maximise health gain with constrained health sector resources, it is necessary to identify the highest priority risk factor areas for studying preventive interventions. We aimed to develop a process for prioritising such risk factors for further research, in the New Zealand setting.

Methods Using WHO data for high-income countries in the Western Pacific Region, the burden of disease in disability-adjusted life years associated with leading risk factors was used as a starting point. Subsequent prioritising steps included: the existence of effective and (likely) cost-effective preventive interventions for each risk factor, and the contribution of the risk factor to health inequalities.

Results The top six major risk factors identified were: tobacco use, high blood pressure, high cholesterol, alcohol use, overweight/ obesity and physical inactivity. All of these six risk factors contribute to ethnic health inequalities (Maori vs non-Maori). They are also all relevant to reducing the health burden for children/youth and older adults, and four were relevant to reducing health inequalities for socio-economically deprived New Zealanders. For all of the top six risk factor areas there are published studies indicating that one or more preventive interventions are cost-saving (to the health sector or society).

Conclusions This process identified risk factor areas associated with high health burden and which are amenable to cost-effective preventive interventions. Our next step is to work with stakeholders to select the range of interventions within each risk factor area that are of most interest for cost-effectiveness analysis.
Introduction There are several population-based English studies of AMI, but they are local rather than national, do not necessarily distinguish first events and re-infarctions, and are out of date. Our objective was to study AMI incidence and 30-day case fatality rates (CFRs) for first and recurrent events in England in 2007 (population 51.8 million).

Methods Analysis of linked routine national hospital and mortality data, with an 8-year washout period to identify people who already had a prior event, in order to distinguish whether each AMI in 2007 was first or recurrent.

Results Of 88311 AMI events in 2007, 86\% were first events. Agestandardised incidence of first AMI (per 100000 person years) was 151 (150-152) in men and 64 (63-65) in women. Age-standardised 30-day overall CFRs including sudden deaths without hospitalisation for first AMI were 35.5\% (35.1-36.0) and 34.4\% (33.3-35.4) in men and $34.4 \%(33.9-35.0)$ and $31.0 \%$ (29.8-32.3) in women, for first AMIs and re-infarctions, respectively. Age-standardised 30-day hospitalised CFRs were much lower at $14.5 \%(14.1-14.9)$ and $14.1 \%$ $(13.2-15.0)$ in men and $15.2 \%(14.7-15.7)$ and $13.9 \%(12.8-15.0)$ in women for first AMIs and re-infarctions, respectively.

Conclusions This study provides, for the first time, total nationallevel population-based estimates of first and recurrent AMI as well as overall AMI incidence and case fatality in England. It holds informative value in better understanding AMI epidemiology and prognosis that should prove useful to practitioners and policy makers.

\section{P2-334 ALCOHOL AND MORTALITY AMONG 220000 MEN IN CHINA 1990-2005: A NATIONALLY REPRESENTATIVE PROSPECTIVE STUDY}

doi:10.1136/jech.2011.142976k.66

${ }^{1} \mathrm{~L}$ Yang, ${ }^{*}{ }^{2} \mathrm{M}$ Zhou, ${ }^{1} \mathrm{P}$ Sherliker, ${ }^{2} \mathrm{G}$ Yang, ${ }^{1} \mathrm{R}$ Peto, ${ }^{2} \mathrm{~J}$ Wang, ${ }^{1} \mathrm{I}$ Millwood, ${ }^{2} \mathrm{Y} \mathrm{Hu}$, ${ }^{1} \mathrm{M}$ Smith, ${ }^{1} \mathrm{Z}$ Chen. ${ }^{1}$ University of Oxford, Oxford, UK; ${ }^{2}$ Chinese Center for Disease Control and Prevention, Beijing, China

Introduction Light-to-moderate alcohol consumption is associated with decreased mortality from cardiovascular diseases in most studies in the West. Data on the health effects of alcohol drinking in China, which involves mainly distilled spirit, are limited.

Methods A nationally representative prospective cohort study included 220000 Chinese men aged 40-79 years who were recruited in 1990-1991 from 45 widely separated study sites. By 1 January 2006, over 40000 deaths had been recorded at age 40-80 years. Cox regression was used to relate alcohol drinking to cause-specific mortality, adjusting simultaneously for age, area, smoking and education.

Results About a third of participants reported drinking alcohol regularly (ie, drank at least once a week), mainly involving spirit. The estimated mean alcohol consumption among regular drinkers was $372 \mathrm{~g} /$ week. There was a J-shaped association between amount of alcohol consumed and all-cause mortality, after excluding all men with prior disease at baseline and the first 3 years of follow-up, with those who drank $<140 \mathrm{~g} /$ week having the lowest overall mortality. Regular alcohol drinking was hardly related to respiratory mortality, but J-shaped associated with mortality from IHD, stomach cancer and lung cancer, and positively associated with mortality from stroke, oesophageal cancer and liver cirrhosis and
K Smolina, ${ }^{*}$ M Goldacre, L Wright, M Rayner. University of Oxford, Oxford, UK 